\title{
PERFIL FUNCIONAL DE COMUNICAÇÃO E DESEMPENHO SÓCIO-COGNITIVO DE ADOLESCENTES AUTISTAS INSTITUCIONALIZADOS
}

\section{Functional communicative profile and social-cognitive performance of institutionalized autistic adolescents}

\author{
Danielle Azarias Defense (1), Fernanda Dreux Miranda Fernandes ${ }^{(2)}$
}

\begin{abstract}
RESUMO
Objetivo: o objetivo dessa pesquisa foi descrever as características funcionais da comunicação de adolescentes autistas institucionalizados, ao longo de um período de aproximadamente seis meses, nos aspectos de: número de atos comunicativos por minuto, proporção de interpessoalidade da comunicação e proporção de utilização dos meios comunicativos gestual, vocal e verbal assim como descrever as características do desempenho sócio-cognitivo dos sujeitos ao longo desse período. Métodos: foram sujeitos dessa pesquisa oito adolescentes autistas institucionalizados que foram avaliados durante seis meses quanto ao perfil funcional de comunicação e ao desempenho sóciocognitivo. Os dados obtidos foram submetidos à análise estatística não paramétrica. Resultados: foi observada evolução quanto ao número de atos comunicativos por minuto e à proporção de funções comunicativas interpessoais, mas não foi observada evolução significativa quanto aos aspectos sócio-cognitivos. Conclusão: foi possível observar evolução na linguagem em adolescentes autistas institucionalizados num período de tempo de seis meses, mas não foi possível identificar correlações com o desempenho sócio-cognitivo.
\end{abstract}

DESCRITORES: Transtorno Autístico; Adolescente; Linguagem; Cognição

\section{INTRODUÇÃO}

O autismo é uma síndrome comportamental, de etiologia desconhecida e caracterizada por um prejuízo na tríade socialização, linguagem e comportamento. O diagnóstico é feito com a utilização de escalas diagnósticas: o Código Internacional de Doenças (CID 10) ${ }^{1}$ e o Manual de diagnóstico e estatística de distúrbios mentais (DSM IV) ${ }^{2}$. A intensidade dos sintomas do autismo varia de acordo com o nível de desenvolvimento intelectual ou cognitivo.

(1) Fonoaudióloga; Mestranda em Ciências da Reabilitação, Área de Concentração Comunicação Humana da Faculdade de Medicina da Universidade de São Paulo, São Paulo, SP, Brasil.

(2) Fonoaudióloga; Professor Associado do Curso de Fonoaudiologia da Faculdade de Medicina da Universidade de São Paulo, São Paulo, SP, Brasil.

Conflito de interesses: inexistente
As relações entre os déficits cognitivos e de linguagem nos quadros de autismo infantil têm sido amplamente discutidas na literatura ${ }^{3-7}$.

As pesquisas com adolescentes autistas 8,9 mostraram que estes apresentam dificuldades de imitação e que a severidade da disfunção sensorial não está associada à severidade dos sintomas ${ }^{9}$. Outro estudo ${ }^{10}$ relatou também a maior ocorrência de prejuízos na comunicação verbal e não verbal em relação às dificuldades de reciprocidade social e à diminuição de comportamentos repetitivos nessa faixa etária. Em relação ao desenvolvimento, os estudos desenvolvidos têm observado que os autistas melhoram nas funções executivas entre as idades de 11 e 16 anos ${ }^{3,11,12}$.

A necessidade de pesquisas que investiguem a linguagem considerando os aspectos cognitivos é citada em diversos estudos sobre autismo há várias décadas ${ }^{4,13-19}$ assim como outras pesquisas ${ }^{3,11,12}$ abordam mudanças nesses aspectos o longo do 
tempo, apontando para a necessidade de estudos com adolescentes e adultos autistas para melhor caracterização de seus sintomas e da evolução observada no desenvolvimento desses.

Diante dos aspectos descritos acima, o objetivo dessa pesquisa foi descrever as características funcionais da comunicação de adolescentes autistas institucionalizados, ao longo de um período de aproximadamente seis meses, nos aspectos de: número de atos comunicativos por minuto, proporção de interpessoalidade da comunicação e proporção de utilização dos meios comunicativos gestual, vocal e verbal assim como descrever as características do desempenho sócio-cognitivo dos sujeitos ao longo de um período de aproximadamente seis meses.

As hipóteses sugeridas em relação ao objetivo proposto advêm da base teórica compilada e da própria prática clínica: haverá desenvolvimento observável e significativo no perfil funcional da comunicação, no desempenho sócio-cognitivo num período de seis meses e o desenvolvimento sóciocognitivo será mais evidente do que o da linguagem no período estudado.

\section{MÉTODOS}

O local de realização da pesquisa foi um Centro de Convivência conveniado à Secretaria da Saúde do Estado de São Paulo que atende pacientes portadores de autismo com ou sem co-morbidades e de diversas faixas etárias. Todos os responsáveis dos sujeitos envolvidos na pesquisa assim como o responsável da instituição assinaram o Termo de Consentimento Livre e Esclarecido.

Os critérios de inclusão no estudo foram: estar na faixa etária da adolescência, freqüentar regularmente o Centro de Convivência, participar de oficina de linguagem, apresentar quadro de autismo sem co-morbidades e laudo médico de autismo de acordo com o DSM-IV 2 ou da CID-10 ${ }^{1}$. Seguindo esses critérios, apenas oito sujeitos, entre 12 e 15 anos, foram selecionados para fazer parte da pesquisa. Portanto, o número pequeno de sujeitos do estudo justifica-se pela busca de maior homogeneidade.

O material utilizado foram os protocolos para o Perfil Funcional da Comunicação ${ }^{20}$ e Teste de Desempenho Sócio-Cognitivo ${ }^{21}$, incluindo o material específico, DVDs, filmadoras e computador.

Os procedimentos para a coleta de dados envolveram três filmagens, de 15 minutos, com intervalos aproximados de três meses entre elas, para cada sujeito.
Para a investigação do Perfil Funcional da Comunicação ${ }^{20}$, as filmagens foram feitas em situações de interação espontânea, na situação rotineira de almoço, em interação com a pesquisadora (familiar aos sujeitos e ao contexto). A escolha da situação de interação espontânea, durante atividade de vida diária, ocorreu buscando garantir a homogeneidade da situação de coleta de dados, evitar interferência na rotina diária dos sujeitos e aproveitar a familiaridade da terapeuta com os sujeitos e com o contexto. As variáveis determinadas para análise foram: atos comunicativos, meio comunicativo e funções comunicativas.

O procedimento para a verificação do Desempenho Sócio - Cognitivo ${ }^{21}$ envolveu duas situações: espontânea e de teste. Para a análise da situação espontânea foram utilizadas as mesmas filmagens usadas para a análise da comunicação. A aplicação do teste ocorreu sempre no mesmo dia em que as situações espontâneas foram filmadas e foi realizada sempre numa sala de atendimento individual familiar para os sujeitos.

Todos os dados obtidos foram registrados em protocolos individuais específicos, transcritos para a planilha de dados e submetidos a análise estatística.

A pesquisa foi aprovada pela Comissão Ética em Pesquisa do Hospital das Clínicas da Faculdade de Medicina da Universidade de São Paulo, com o protocolo número 382/04.

Os dados foram submetidos a análise estatística que usou testes não - paramétricos (Friedman, Wilcoxon e Qui-quadrado, com nível de significância de $5 \%$ ), por se tratar de uma amostra pequena.

Além disso, o teste de Correlação de Spearman foi aplicado para avaliar as associações entre todas as variáveis deste estudo quanto a relação positiva e a negativa. A partir desses cálculos podemse verificar os valores do coeficiente de correlação (rho) de Pearson que verifica se a força da associação e se ela é significante ( $p$-valor). Os coeficientes de correlação indicam a força da relação entre duas variáveis e foram interpretados ${ }^{22}$.

\section{RESULTADOS}

As Tabelas de 1 a 5 apresentam os resultados da análise do Perfil Funcional de Comunicação, onde observou-se aumento no total de atos comunicativos e dos atos comunicativos por minuto a partir da segunda coleta e que se manteve na terceira. $O$ meio comunicativo predominante foi o gestual e as funções foram as não interpessoais. 
Tabela 1 - Média, mediana e desvio padrão do número de atos comunicativos produzidos por todos os sujeitos em cada um dos momentos de coleta de dados e comparação entre as três coletas quanto ao número de atos comunicativos por minuto - teste de Spearman

\begin{tabular}{|c|c|c|c|c|c|c|c|}
\hline & & média & mediana & DP & $\mathrm{x}^{2}$ & $p$-valor & \\
\hline $\begin{array}{c}1^{\underline{a}} \\
\text { COLETA }\end{array}$ & Total & 42,38 & 43,50 & 27,18 & & & \\
\hline & Atos/min & 2,84 & 2,89 & 1,79 & & & \\
\hline$\stackrel{2^{\mathrm{a}}}{\mathrm{COIFTA}}$ & Total & 96,4 & 93,5 & 50,1 & & & \\
\hline & Atos/min & 6,65 & 6,36 & 3,41 & & & \\
\hline $\begin{array}{c}3^{\underline{a}} \\
\text { COLETA }\end{array}$ & Total & 102,5 & 108,0 & 31,5 & & & \\
\hline & Atos/min & 6,6 & 6,56 & 1,99 & & & \\
\hline COLETAS & Atos/min & & & & 14,250 & 0,001 & $\begin{array}{c}\text { Coleta } 1< \\
\text { coleta } 2 \text { e } \\
\text { coleta } 3\end{array}$ \\
\hline
\end{tabular}

Total = total de atos comunicativos de todos os sujeitos em cada coleta.

Atos $/ \mathrm{min}=$ número de atos comunicativos de todos os sujeitos em cada coleta.

DP = desvio padrão

$\mathrm{X}^{2}=$ Teste de Spearman

$\mathrm{p}$-valor $=0,001$

Os testes estatísticos utilizados foram: média, mediana, desvio padrão e Teste de Spearman.

Tabela 2 - Meio comunicativo predominante para cada coleta e comparação entre as três coletas para cada meio comunicativo - teste de Friedman e Wilcoxon

\begin{tabular}{lcccccccc}
\hline & Média & Mediana & DP & Friedman & Wilcoxon & & $\mathbf{X}^{2}$ & p-valor \\
\hline GE1 & 64,67 & 62,98 & 23,45 & $\mathrm{X}^{2}=7,548 ;$ & GE $>$ VO & GE & 1,355 & 0,508 \\
VO1 & 10,89 & 10,13 & 10,30 & $\mathrm{p}=0,023$ & & & & \\
VE1 & 24,44 & 15,70 & 27,84 & & & & \\
GE2 & 73,23 & 68,15 & 13,66 & $\mathrm{X}^{2}=13,067 ;$ & $\mathrm{GE}>\mathrm{VO}, \mathrm{VE}$ & $\mathrm{VO}$ & 0,452 & 0,798 \\
VO2 & 13,84 & 13,08 & 11,17 & $\mathrm{p}=0,001$ & & & & \\
VE2 & 12,93 & 10,63 & 13,99 & & & & \\
GE3 & 75,43 & 78,02 & 14,68 & $\mathrm{X}^{2}=12,000 ;$ & $\mathrm{GE}>\mathrm{VO}, \mathrm{VE}$ & $\mathrm{VE}$ & 2,800 & 0,247 \\
VO3 & 12,06 & 10,03 & 10,36 & $\mathrm{p}=0,002$ & & & & \\
VE3 & 12,38 & 13,87 & 11,60 & & & & & \\
\hline
\end{tabular}

$\mathrm{GE}=$ meio gestual

$\mathrm{VO}=$ meio vocal

$\mathrm{VE}=$ meio verbal

$1=$ primeiro momento da coleta

2 = segundo momento da coleta

3 = terceiro momento da coleta

$\mathrm{DP}=$ desvio padrão

$\mathrm{X}^{2}=$ Teste de Friedman

Os testes estatísticos utilizados foram: média, mediana, desvio padrão, Friedman e Wilcoxon. 
Tabela 3 - Porcentagem de funções interpessoais e não interpessoais em cada coleta

\begin{tabular}{ccccccc}
\hline SUJEITOS & \multicolumn{2}{c}{ \% FUNÇÕES INTERPESSOAIS } & \multicolumn{3}{c}{ \% FUNÇÕES NÃO INTERPESSOAIS } \\
\hline NOME & $\mathbf{1}^{\mathbf{a}}$ coleta & $\mathbf{2}^{\mathbf{a}}$ coleta & $\mathbf{3}^{\mathbf{a}}$ coleta & $\mathbf{1}^{\text {a }}$ coleta & $\mathbf{2}^{\mathbf{a}}$ coleta & $\mathbf{3}^{\mathbf{a}}$ coleta \\
\hline MF & 13,04 & 19,32 & 23,47 & 86,96 & 80,68 & 76,53 \\
LP & 28,77 & 5,45 & 18,64 & 71,23 & 94,55 & 81,36 \\
EL & 81,81 & 63,15 & 42,86 & 18,18 & 35,85 & 57,14 \\
LO & 43 & 34 & 54,36 & 57 & 66 & 45,64 \\
EB & 42,30 & 52,17 & 29,85 & 57,70 & 47,83 & 70,15 \\
GB & 76,08 & 33 & 36,36 & 23,92 & 67 & 63,64 \\
CH & 43,90 & 40 & 46,61 & 56,09 & 60 & 53,39 \\
GA & 40 & 30,59 & 40,23 & 60 & 69,41 & 59,77 \\
\hline
\end{tabular}

$\%$ FUNCÕES INTERPESSOAIS = porcentagem de funções comunicativas interpessoais.

\% FUNÇÕES NÃO INTERPESSOAIS = porcentagem de funções comunicativas não interpessoais.

Tabela 4 - Comparação entre as três coletas quanto á ocorrência de funções interpessoais - teste qui-quadrado

\begin{tabular}{|c|c|c|c|c|c|}
\hline & \multicolumn{2}{|c|}{ Interpessoal } & \multicolumn{2}{|c|}{ Não interpessoal } & \multirow[t]{2}{*}{ TOTAL } \\
\hline & $f$ & $\%$ & $f$ & $\%$ & \\
\hline $1^{\text {a }}$ coleta & 141 & 41,59 & 198 & 58,41 & 339 \\
\hline $2^{\mathrm{a}}$ coleta & 217 & 28,14 & 554 & 71,85 & 771 \\
\hline \multirow[t]{2}{*}{$3^{\mathrm{a}}$ coleta } & 302 & 36,83 & 518 & 63,17 & 820 \\
\hline & \multicolumn{5}{|c|}{$X^{2}=23,314 ;$ n.g.I. $=2 ; p<0,001$} \\
\hline
\end{tabular}

Interpessoal = funções comunicativas interpessoais

Não - interpessoal $=$ funções comunicativas não interpessoais

$\mathrm{f}=$ número de funções

$\%=$ porcentagem

TOTAL = total de funções comunicativas interpessoais e não interpessoais

Tabela 5 - Correlação de Spearman entre as variáveis do perfil funcional da comunicação

\begin{tabular}{cccccc}
\hline p-valor (rho) & Atos 1 & Atos 2 & Atos 3 & Inter 1 & Inter 2 \\
\hline \multirow{2}{*}{ Inter 1 } & 0,407 & 0,459 & 0,347 & & \\
& $(-0,342)$ & $(-0,307)$ & $(-0,385)$ & & \\
Inter 2 & 0,126 & $0,007^{* *}$ & 0,125 & $0,067^{*}$ & \\
& $(-0,588)$ & $(-0,857)^{* *}$ & $(-0,589)$ & $(0,674)^{*}$ & \\
Inter 3 & 0,977 & 0,363 & 0,533 & 0,247 & 0168 \\
& $(-0,012)$ & $(-0,373)$ & $(0,261)$ & $(0,464)$ & $(0,539)$ \\
\hline
\end{tabular}

inter $=$ atos comunicativos com funções interpessoais

Atos $=$ atos comunicativos

$1=$ primeiro momento da coleta

2 = segundo momento da coleta

$3=$ terceiro momento da coleta

Rho= correlação de Pearson

** = associação positiva ou negativa alta ou muito alta.

* = associação moderada positiva ou negativa.

Correlação de Spearman 
As Tabelas 6 e 7 sintetizam os resultados da análise do Desempenho Sócio-Cognitivo dos sujeitos deste estudo nas duas situações, teste e espontânea, nos três momentos de coleta. Observou-se que na terceira coleta na situação espontânea obteve uma maior pontuação dos sujeitos e que no desempenho geral do grupo em situação espontânea houve uma evolução num período de seis meses.

A tabela 6 apresenta a análise das correlações entre as variáveis do desempenho sócio-cognitivo, momento de coleta de dados e situação espontânea e teste.

A partir desses dados pode-se observar associações fortes (coeficientes altos e muito altos) e positivas no sentido de que:
1) Quanto maior o escore de desempenho sóciocognitivo na situação teste 1, maior foi o escore do desempenho nas situações teste e espontânea 2 e 3.

2) Quanto maior o escore de desempenho sóciocognitivo na situação teste 3 , maior foi o escore de desempenho na situação espontânea 3.

3) Quanto maior o escore de desempenho sóciocognitivo na situação espontânea 1, maior foi o escore de desempenho na situação espontânea 3.

4) Quanto maior o escore de desempenho sóciocognitivo na situação espontânea 2, maior foi o escore de desempenho na situação espontânea 3.

Tabela 6 - Desempenho sócio-cognitivo em situação de teste e espontâneo - pontuação total de cada sujeito em cada coleta

\begin{tabular}{ccccccc}
\hline & \multicolumn{2}{c}{ 1 $^{\mathbf{a}}$ COLETA } & \multicolumn{2}{c}{ 2 $^{\mathbf{a}}$ COLETA } & \multicolumn{2}{c}{ 3 $^{\mathbf{a}}$ COLETA } \\
\hline NOME & Teste & Espontâneo & Teste & Espontâneo & Teste & Espontâneo \\
\hline MF & 15 & 16 & 17 & 14 & 23 & 21 \\
LP & 23 & 26 & 21 & 20 & 31 & 26 \\
EL & 7 & 15 & 11 & 14 & 4 & 17 \\
LO & 16 & 25 & 19 & 18 & 15 & 27 \\
EB & 23 & 21 & 13 & 24 & 26 & 28 \\
GB & 28 & 25 & 27 & 28 & 29 & 27 \\
CH & 20 & 27 & 22 & 33 & 27 & 32 \\
GA & 24 & 21 & 26 & 26 & 27 & 29 \\
\hline
\end{tabular}

Teste $=$ dados coletados na situação de teste

Espontâneo = dados coletados na situação espontânea

Tabela 7 - Comparação entre as situações teste e espontânea de investigação do desempenho sóciocognitivo em cada momento de coleta entre as coletas nas situações teste e espontânea - testes de Friedman e Wilcoxon

\begin{tabular}{lccccccc}
\hline & & média & mediana & DP & Z Wilcoxon & p-valor & $\mathbf{X}^{\mathbf{2}}$ \\
\hline 1ª $^{\text {a }}$ COLETA & Teste & 19,50 & 21,50 & 6,61 & $-1,126$ & 0,260 & \\
& Espontânea & 22,00 & 23,00 & 4,57 & & & \\
$2^{\text {a }}$ COLETA & Teste & 19,50 & 20,00 & 5,71 & $-0,940$ & 0,347 & \\
& Espontânea & 22,13 & 22,00 & 6,81 & & & \\
$3^{\text {a }}$ COLETA & Teste & 22,75 & 26,50 & 8,99 & $-1,065$ & 0,287 & \\
& Espontânea & 25,88 & 27,00 & 4,73 & & $\mathbf{0 , 1 9 7}$ & $\mathbf{3 , 2 5 0}$ \\
& Teste & & & & Coleta 3 > coleta & $\mathbf{0 , 0 5 0}$ & $\mathbf{6 , 0 0 0}$ \\
COLETAS & Espontânea & & & & & & \\
\hline
\end{tabular}

Teste $=$ dados coletados na situação de teste

Espontâneo = dados coletados na situação espontânea

$Z$ = Wilcoxon

$\mathrm{DP}=$ desvio padrão

$\mathrm{X}^{2}=$ Friedman

Os testes estatísticos usados foram: Média, Mediana, Desvio Padrão, Wilcoxon e Friedman. 
A Tabela 5 apresenta a análise de correlação entre o número de atos comunicativos produzidos por minuto e as funções interpessoais nas três situações de coleta de dados.

A Tabela 8 apresenta a análise de correlação entre os escores de desempenho sócio-cognitivo e as variáveis de atos comunicativos por minuto e atos comunicativos com funções interpessoais.
Os resultados revelam associações apenas moderadas entre o primeiro momento de coleta de dados em situação espontânea e o número de atos comunicativos produzidos por minuto no segundo e no terceiro momento de coleta de dados. A outra associação, também moderada, refere-se à proporção de atos comunicativos com funções interpessoais e o desempenho sócio-cognitivo na terceira situação de teste.

Tabela 8 - Correlação de Spearman entre o desempenho sócio-cognitivo e as variáveis do perfil funcional da comunicação nos três momentos de coleta de dados

\begin{tabular}{ccccccc}
\hline $\begin{array}{c}\text { p-valor } \\
\text { (rho) }\end{array}$ & Teste 1 & Teste 2 & Teste 3 & Espont.1 & Espont. 2 & Espont. 3 \\
\hline Atos1 & 0,802 & 0,684 & 0,682 & 0,139 & 0,733 & 0,678 \\
& $(0,107)$ & $(0,172)$ & $(0,173)$ & $(0,571)$ & $(-0,145)$ & $(0,175)$ \\
Atos 2 & 0,222 & 0,131 & 0,112 & $0,060^{*}$ & 0,641 & 0,429 \\
& $(0,486)$ & $(0,581)$ & $(0,605)$ & $(0,686)^{*}$ & $(0,197)$ & $(0,327)$ \\
Atos 3 & 0,634 & 0,250 & 0,523 & $0,051^{*}$ & 0,726 & 0,251 \\
& $(0,201)$ & $(0,461)$ & $(0,267)$ & $(0,704)^{*}$ & $(0,148)$ & $(0,461)$ \\
Inter 1 & 0,764 & 0,916 & 0,253 & 0,894 & 0,745 & 0,596 \\
& $(-0,127)$ & $(-0,045)$ & $(-0,458)$ & $(-0,057)$ & $(0,138)$ & $(-0,223)$ \\
Inter 2 & 0,290 & 0,168 & $0,088^{*}$ & 0,353 & 0,949 & 0,611 \\
& $(-0,428)$ & $(-0,539$ & $(-0,639) *$ & $(-0,380)$ & $(0,027)$ & $(-0,214)$ \\
Inter 3 & 0,522 & 0,866 & 0,215 & 0,671 & 0,590 & 0,615 \\
& $(-0,267)$ & $(0,072)$ & $(-0,492)$ & $(0,179)$ & $(0,226)$ & $(0,212)$ \\
\hline
\end{tabular}

* associação moderada positiva ou negativa.

inter $=$ atos comunicativos com funções interpessoais

Atos $=$ atos comunicativos

Teste $=$ Dados coletados na situação de teste

Espont $=$ Dados coletados na situação espontânea

1 = primeiro momento da coleta

2 = segundo momento da coleta

$3=$ terceiro momento da coleta

rho= correlação de Pearson

Correlação de Spearman

\section{DISCUSSÃO}

Nos resultados obtidos no Perfil Funcional de Comunicação, observou-se um aumento no número do total de atos comunicativos na segunda coleta de dados, em relação à primeira, e este resultado se manteve na terceira coleta. As funções não interpessoais são mais freqüentes na segunda coleta do que na primeira e terceira coletas, que diferem estatisticamente. O meio de comunicação de maior predominância foi o gestual e as funções não interpessoais predominaram nos três momentos de coleta.

Os achados acima estão de acordo com estudo anterior ${ }^{3}$ que observaram indivíduos autistas entre 10 e 15 anos de idade, demonstrando que estes podem apresentar melhoras na interação e nas habilidades de linguagem. Em relação ainda aos achados acima, podemos citar outro estudo ${ }^{23}$ que identificou maiores dificuldades de comunicação nos indivíduos autistas adolescentes e adultos.

Essas observações exigem reflexão a respeito da importância da terapia fonoaudiológica no desenvolvimento da linguagem de autistas de diversas faixas etárias e principalmente da necessidade da continuidade de atendimento fonoaudiológico aos sujeitos autistas adolescentes.

As grandes diferenças individuais observadas nos resultados referentes ao Perfil Funcional de Comunicação em situação espontânea estão de acordo com achados de estudos anteriores 18, 24. Em outra pesquisa ${ }^{25}$ há a afirmação que os indivíduos autistas apresentam mais respostas em ambientes naturais e estas variam em função e 
forma comunicativa; por isso os sujeitos estudados foram observados em situação espontânea visando um ambiente naturalista para a coleta de dados que, por outro lado, não determinou menor variação nas funções comunicativas utilizadas ou nos meios comunicativos usados para expressá-las.

Outro aspecto importante a ser observado é que esses sujeitos apresentaram evolução da primeira para a segunda coleta de dados em relação ao número de atos comunicativos. Isso pode devido à exposição a situação de comunicação com pares, que proporcionam situações espontâneas em que estes indivíduos são obrigados a se comunicar com seus colegas e com o terapeuta, da forma mais eficiente possível.Este achado está de acordo com o trabalho desenvolvido anteriormente ${ }^{26}$ a respeito de três modelos diferentes de situação terapêutica, que demonstrou que os sujeitos que eram atendidos em oficinas de linguagem apresentaram maior evolução no número de atos comunicativos do que nas abordagens de terapia individual e terapia individual realizada juntamente com a mãe.

Logo, considerando os achados dessa pesquisa a respeito do perfil funcional de comunicação observa-se que estes se encontram de acordo com pesquisas já desenvolvidas e demonstraram confirmação parcial da hipótese de que haveria desenvolvimento observável e significativo no perfil funcional da comunicação num período de seis meses.

Quanto ao desempenho sócio-cognitivo ${ }^{21}$, os resultados não se diferenciam estatisticamente nas três coletas; porém na terceira coleta, em situação espontânea, os sujeitos tiveram maior pontuação individualmente, apesar de não terem obtido evolução como um grupo, fato esse que também pode ser justificado devido às diferenças individuais encontradas nos quadros de autismo.

A possibilidade de um melhor desempenho sócio-cognitivo em alguns indivíduos está de acordo com a afirmação de estudo anterior ${ }^{12}$ de que os autistas melhoram em funções executivas ao longo do tempo entre as idades de 11 e 16 anos.

O fato de que a evolução no desempenho sóciocognitivo foi observada na situação espontânea, corrobora a afirmação de pesquisador ${ }^{25}$, no sentido de que as situações naturais tendem a produzir os melhores dados de investigação a respeito do desempenho de indivíduos autistas.

O desempenho sócio- cognitivo estudado nessa pesquisa demonstrou a confirmação parcial da hipótese de desenvolvimento observável e significativo no período estudado e aponta para as questões de heterogeneidade dos sujeitos com autismo e a questão da familiaridade e naturalidade de situações em que são expostos favorecerem melhores resultados.
O presente estudo buscou resultados em relação ao desempenho sócio-cognitivo e à linguagem e a inter-relação entre esses, baseado na afirmação da pesquisa ${ }^{19}$ que comentou da necessidade de se levar em consideração a inter-relação entre os elementos cognitivos no desenvolvimento da linguagem.

A evolução observada em alguns aspectos do perfil funcional da comunicação e do desempenho sócio-cognitivo dessa pesquisa está de acordo com outro estudo ${ }^{27}$ que concluiu que as mudanças no desempenho sócio-cognitivo e no perfil funcional da comunicação podem ser consideradas interligadas, mas não lineares.

Os resultados corroboram também a afirmação de outra pesquisa ${ }^{19}$ no sentido de que há poucas correlações estatisticamente significantes entre aspectos sócio-cognitivos e o perfil funcional de comunicação de indivíduos com autismo.

Considerando os resultados do perfil funcional da comunicação e desempenho sócio-cognitivo, a hipótese do desenvolvimento sócio-cognitivo ser mais evidente do que na linguagem foi negada, visto que os resultados demonstraram mais dados observáveis do desenvolvimento da linguagem.

Por fim, observou-se que os adolescentes autistas institucionalizados participantes dessa pesquisa apresentaram evolução na linguagem nesse modelo como já foi encontrado em pesquisa anterior ${ }^{16}$.

\section{CONCLUSÃO}

O presente estudo propôs a verificação de três hipóteses e as conclusões referem-se à amostra estudada:

A primeira hipótese afirmava que "haverá desenvolvimento observável e significativo no perfil funcional da comunicação num período de seis meses". Essa hipótese foi parcialmente confirmada, na medida em que apenas os resultados referentes ao número de atos comunicativos e à proporção de funções comunicativas interpessoais evidenciaram evolução estatisticamente significativa, quando analisados os dados de todo o grupo.

A segunda hipótese era de que "haverá desenvolvimento observável e significativo no desempenho sócio-cognitivo em um período de seis meses" também foi apenas parcialmente confirmada, pois a diferença estatisticamente significativa foi detectada apenas para o terceiro momento de coleta de dados, em situação espontânea.

A confirmação parcial das duas primeiras hipóteses nos remeteu à questão de que foi possível observar evolução nos aspectos da linguagem e 
sócio-cognitivos em indivíduos autistas na faixa etária da adolescência.

Uma terceira hipótese foi proposta, no sentido de que "o desenvolvimento sócio-cognitivo será mais evidente do que o da linguagem nessa pesquisa". Essa hipótese foi negada, na medida em que foram identificados mais dados significativos de evolução no que se refere ao perfil funcional da comunicação do que ao desempenho sócio-cognitivo.

Esses dados reafirmam a grande dificuldade em se traçar um perfil fenotípico único para sujeitos do espectro autístico, devido à grande heterogeneidade do quadro.

\section{AGRADECIMENTOS}

Agradeço primeiramente a Profa. Dra. Fernanda Dreux Miranda Fernandes pela oportunidade, apoio e aprendizado. Agradeço também ao local aonde realizei a pesquisa, aos pais e adolescentes autistas que participaram dela e aos meus familiares que sempre me apoiaram.

\begin{abstract}
Purpose: this study aims at describing the functional communication characteristics of institutionalized autistic adolescents for a period of six months approximately as for: number of communicative acts per minute, interpersonal proportion and means of proportion communication use (gestural, vocal, verbal) as well as to describe the subjects' characteristics as for social cognitive performance in this period. Methods: the subjects were eight institutionalized adolescents with autism. Their functional communicative profile and social-cognitive performance were assessed during a six-month period and resulting data were analyzed with non-parametric statistic methods. Results: it was possible to observe that there was an improvement in the number of communicative acts per minute and in the proportion of interpersonal communicative functions. No significant change was observed as for the social-cognitive performance. Conclusion: it was possible to observe evolution in the language of institutionalized adolescents with autism during a six-month period, but it was not possible to identify its correlation with their social-cognitive performance.
\end{abstract}

KEYWORDS: Autistic Disorder; Adolescent; Language; Cognition

\section{REFERÊNCIAS}

1. OMS (Organização Mundial e Saúde). Classificação de Transtornos Mentais e de Comportamento do CID 10. Porto Alegre: Artes Médicas; 1993.

2. Americam Psychiatric Association (APA). Manual de diagnóstico e estatístico de distúrbios mentais. DSM-IV. São Paulo: Manole;1994.

3. Millher LP, Fernandes FDM. Habilidades pragmáticas, vocabulares e gramaticais em crianças com transtornos do espectro autístico. Pró-Fono, 2009; 21: 309-14

4. Fernandes FDM. Resultados de terapia fonoaudiológica com adolescentes com diagnóstico inserido no espectro autístico. Pró-Fono. 2005; 17(1): 67-76.

5. Fernandes FDM. Famílias com crianças autistas na literatura internacional. Rev. soc. bras. fonoaudiol. 2009; 14: 427-32.

6. Bishop DVM, Norbury CF. Executive Functions in Children with communication Impairments, in
Relation to Autistic Symptomatology. Autism. 2005; 9(1): 7-27.

7. Whitehouse AJO, Mayberry MT, Durkin K. Evidence against poor semantic encoding in individuals with autism. Autism. 2007;11(3): 241-54. 8. Freitag CM, Kleser C, Gontardf AV. Imitation and language abilities in adolescents with autism spectrum disorder without language delay. Eur J Child Adolesc Psychiatry. 2006; 15: 282 - 91.

9. Kern JK et al. Sensory correlations in autism. Autism. 2007; 11(2): 123- 34.

10. Shattuck PT et al. Change in Autism Symptoms and Maladaptative Behaviours in Adolescents and Adults with an Autism Spectrum Disorder. J Autism Dev Disord. 2007; 37: 1735- 47.

11. Happé F, Booth R, Charlton, R, Hughes C. Executive function deficits in autism spectrum disorders and attention deficit/ hyperactivity disorder: examinig profiles across domain and ages. Brain Cogn. 2006; 61(1): 25-39.

12. Solomon M, Ozonoff SJ, Cummings N, Carter CS. Cognitive control in autism spectrum disorders. 
University of California; Mind Institute And Imaging Research Center. November, 2007; 239 - 47. 13. Bates E. Language and Context. The aquisition of pragmatics. New York Academic Press; 1976. 14. Fernandes FDM. Refletindo Sobre o Novo Children with autism spectrum disorders - BernardOpitz, Rev. soc. bras. Fonoaudiol. 2009; 13: 148-9. 15. Mechilng LC, Gast DL, Seid NH. Using a personal digital assistant to increase independent task completion by students with autism spectrum disorder. J Autism Dev Disord. 2009, 39(8): 1420-34.

16. Barbosa MRP, Fernandes FDM. Qualidade de vida dos cuidadores de crianças com transtornos do espectro autístico. Rev. soc. bras. Fonoaudiol. 2009; 14: 482-86.

17. Fernandes FDM, Teles $P$. Linguagem nos transtornos do espectro autístico. Rev. soc. bras. fonoaudiol. 2005; 10(4): 207 - 10.

18. Cardoso C, Morato PFS, Andrade S, Fernandes FDM. Desempenho sócio-cognitivo e adaptação sócio-comunicativa em diferentes grupos incluídos no espectro autístico. Pró-Fono. 2010; 22: 43-8.

19. Morato PFS, Fernandes, FDM. Análise do perfil comunicativo quanto à adaptação sóciocomunicativa em crianças do espectro autístico. Rev. soc. bras. fonoaudiol. 2006;11(2): 70-4.

20. Fernandes FDM. Pragmática. In: Andrade CRF, Befi-Lopes DM, Fernandes FDM, Wertzner HF. ABFW - Teste de linguagem infantil nas áreas de fonologia, vocabulário, fluência e pragmática.São Paulo: Ed. Pro-Fono; 2000. p. 77-89.

21. Fernandes FDM, Avejonas DRM, Morato PFS. Perfil funcional da comunicação nos distúrbios do espectro autístico. Revista CEFAC. 2006; 8(1): 20-6.

22. Munro BH. Statistical methods for health care research. In: Specfic statistical tecniques correlation. New York: Lip; 2001. p. 225-43.

23. Chamak B, Bonniau B, Jaunay E, Cohen D. What can we learn about autism from autistic persons? Psychother Psychosom. 2008; 77: 271-9. 24. Fernandes FDM, Miilher LP. Relações entre a Autistic Behavior Checklist (ABC) e o perfil funcional da comunicação no espectro autístico. Pró-Fono R. Atual. Cient. April- June 2008; 20(2): 111-6.

25. Chiang HM. Communicative spontaneity of children with autism. A preliminary analysis. Autism. 2008; 12(1): 9-21.

26. Fernandes FDM, Cardoso C, Sassi FC, Amato CLH, Sousa-Morato PF. Fonoaudiologia e autismo: resultado de três diferentess modelos de terapia de linguagem. Pró-Fono R. Atual. Cient. Oct - Dec 2008; 20(4): 267-72.

27. Cardoso C, Fernandes FDM. Relação entre os aspectos sócio-cognitivos e perfil funcional da comunicação em um grupo de adolescentes do espectro autístico. Pró-Fono R Atual Cient. Jan-Apr. 2006; 18(1): 89-98.
http://dx.doi.org/10.1590/S1516-18462011005000009

RECEBIDO EM: 04/06/2010

ACEITO EM: 13/09/2010

Endereço para correspondência:

Danielle Azarias Defense

Rua Desembargador Euclides de Campos, 46 -

Vila Anglo Brasileira

São Paulo - SP

CEP: 05030-050

E-mail: danielledefense@usp.br 